\title{
Single polarization, dual wavelength fiber laser based on a 3-stage all fiber Lyot filter
}

\author{
Zhijun Yan, Chengbo Mou, Zuxing Zhang, Xiangchuan Wang, Kaimin Zhou and Lin Zhang
}

\begin{abstract}
We have demonstrated a switchable dual-wavelength fiber ring laser with high degree of polarization output by using an intra-cavity 3-stage all fiber Lyot filter. The filter is formed by concatenating four $45^{\circ}$ tilted fiber gratings (45 ${ }^{\circ}$-TFGs) separated by polarization maintaining (PM) fibers with a length ratio of $1: 2: 4(20,40$ and $80 \mathrm{~cm})$, giving a compact integrated configuration with reduced bandwidth. Switchable dual-wavelength or single wavelength output at $1533.5 \mathrm{~nm}$ and $1563.3 \mathrm{~nm}$ has been achieved. The output lasing is considerably stable owing to the in-phase mode-selecting function of the multi-stage Lyot filter, and has a very high degree of polarization higher than $99.9 \%$.

Index Terms - Lyot-filter, tilted fiber grating, fiber ring laser.
\end{abstract}

\section{INTRODUCTION}

A ll fiber stable multi-wavelength and dual-wavelength laser systems have been attracting more interests, because of their wide prospective applications in optical sensing and communications, two-wavelength interferometry, laser spectroscopy, differential absorption lidar, optical data processing and optical instrument testing[1-4]. The erbium-doped fiber amplifiers (EDFAs) have been intensively studied and developed for commercial applications owing to their low cost, high saturation power, low polarization-dependent gain (PDG) and low signal noise ratio in the telecom C-L band region. However, because of relatively broad homogeneous linewidth ( 10nm) at room temperature[5], the EDFA based multi-wavelength laser is always associated with very strong mode competition. So far, the most of the multi-wavelength lasers were achieved by combining an intra-cavity filter with several different techniques include using hybrid gain medium (by adding a semiconductor optical amplifier or Raman amplifier into an EDFA based laser system)[6, 7], using of highly nonlinear configurations[8], inserting a frequency shifter or a phase modulator into the laser cavity [9, 10] and using nonlinear polarization rotation (NPR)[11, 12]. To achieve multi-wavelength operation, an intra-cavity multi-wavelength transmission filter is necessary to be employed, such as in-fiber Mach-Zehnder interferometer[13, 14], Sagnac loop mirror[15-17], birefringent filter[18, 19], sampled and superimposed chirped fiber Bragg

Manuscript received Month XX,XX .. Zhijun Yan (yanz1@aston.ac.uk), Chengbo Mou (c.mou@aston.ac.uk),Zuxing Zhang (z.zhang13@aston.ac.uk), Kaiming Zhou (k.zhou@aston.ac.uk) and Lin Zhang (l.zhang@aston.ac.uk) are with Institute of Photonics and Technologies, School of Engineering and Applied Science, Aston University, Birmingham, UK, B4 7ET.

Xiangchuan Wang (nadazdh@163.com) is with Institute of Optical Communication Engineering, Nanjing University, Nanjing, 210093, China. grating[20, 21] and large angle tilted fiber grating[22]. The Lyot filter is a type of polarization interference filter based on a combination of polarizers and waveplates in alternating sequence, from which a comb-like transmission spectrum could be generated[23, 24]. In our previous work, an all-fiber Lyot filter (AFLF) by sandwiching a segment of PM fiber between two $45^{\circ}$ TFGs UV-inscribed in PM fiber has been reported[25]. The bandwidth and free spectra range (FSR) of a single-stage Lyot filter cannot be separately adjusted by changing the cavity length, because both of the bandwidth and FSR are inversely proportion to the length of the PM fiber, and the bandwidth of Lyot filter is half of the FSR[26]. Fortunately, a multi-stage Lyot filter has the capability of controlling both the bandwidth and FSR separately[27]. For such a multi-stage filter, only the wavelengths which are in phase for each individual stages could have the maximum transmission and the other out-of-phase wavelengths will be suppressed. The bandwidth and FSR of a multi-stage filter is determined by the length of the longest and the shortest cavity, respectively. In this letter, we have proposed and demonstrated a switchable dual-wavelength fiber ring laser by using a 3-stage AFLF based on concatenation of four $45^{\circ}$-TFGs separated by the PM fibers with 1:2:4 length ratio. Comparing with hybrid bulk and fiber birefringence, the multi-stage AFLF features a more compact and integrated structure. Furthermore, the output of the laser is also at high degree of single polarization state.

\section{EXPERIMENT AND RESULTS}

The 3-stage AFLF used in the work was constituted by concatenating four $45^{\circ}$-TFGs with PM fiber cavity length ratio of $1: 2: 4(20 \mathrm{~cm}, 40 \mathrm{~cm}$ and $80 \mathrm{~cm})$. The schematic description of the filter is shown in Fig. 1.

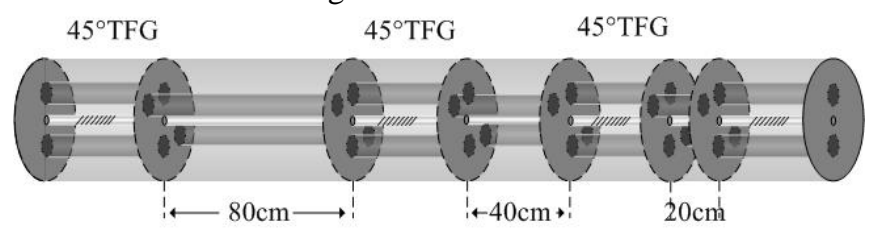

Fig. 1: Schematic structure of a 3-stage AFLF using four $45^{\circ}$-TFGs as in-fiber polarizers.

The transmittance of a multi-stage Lyot filter is given by[27]:

$T=\prod_{i=1}^{m} \cos _{i}^{2}\left(\frac{\pi L_{i} \Delta n}{\lambda}\right) \quad m=1,2,3 \ldots$

Where, $L_{i}$ is the length of $i^{\text {th }} \mathrm{PM}$ fiber cavity; $\Delta n$ is the birefringence of PM fiber; $\lambda$ is the operation wavelength. The 
FSR and bandwidth of a multi-stage Lyot filter can be expressed as:

$$
\begin{aligned}
& F S R \cong \frac{\lambda^{2}}{L_{\text {shortest }} \Delta n} \\
& \Delta \lambda \cong \frac{\lambda^{2}}{2 L_{\text {longest }} \Delta n}
\end{aligned}
$$

Where, $L_{\text {shortest }}$ and $L_{\text {Longest }}$ are the lengths of the shortest and longest PM fiber cavities of the filter.

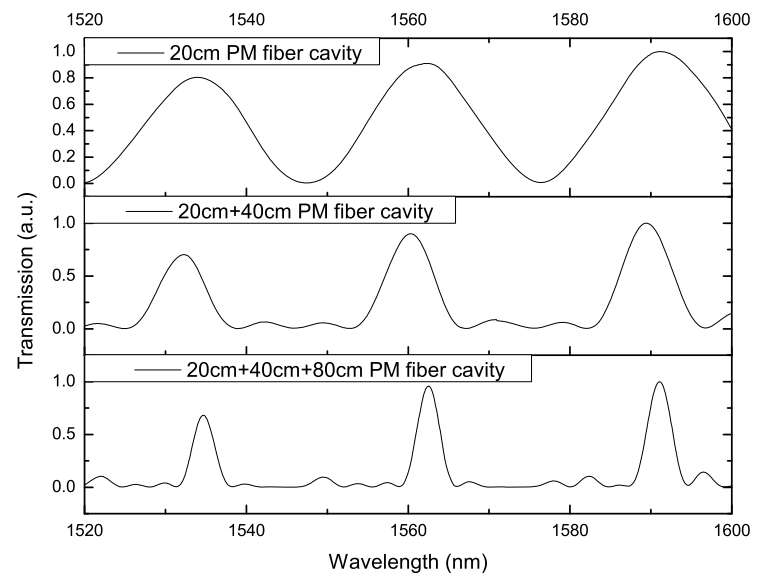

Fig. 2 Measured transmission spectra of three AFLFs with 1-stage $(20 \mathrm{~cm}$ PM fiber cavity); 2-stage (20 $\mathrm{cm}+40 \mathrm{~cm}$ PM fiber cavity) and 3-stage (20 $\mathrm{cm}$ $+40 \mathrm{~cm}+80 \mathrm{~cm}$ PM fiber cavity), respectively.

Fig.2 Error! Reference source not found. shows the transmission spectra of 1-stage, 2-stage and 3-stage AFLFs. It can be seen clearly from the figure that the transmission bands of the AFLFs are generated from coupled cavities, i.e. the transmission maxima are only occur at those wavelengths in phase for each individual cavities and the other non-phase matched wavelengths are suppressed. Meanwhile, we can also see clearly the passband width becomes significantly narrow for multi-stage AFLF. The PM fiber used in our $45^{\circ}$-TFG based AFLFs has a nominal birefringence around $3.27 \times 10^{-4}$. From Equation 2 and 3, the FSR and bandwidth of 3-stage filter we designed are around $26.6 \mathrm{~nm}$ and $6.5 \mathrm{~nm}$, respectively. The 3 -stage filter has two transmission bands at around $1534 \mathrm{~nm}$ and 1564 in the erbium fiber gain range.

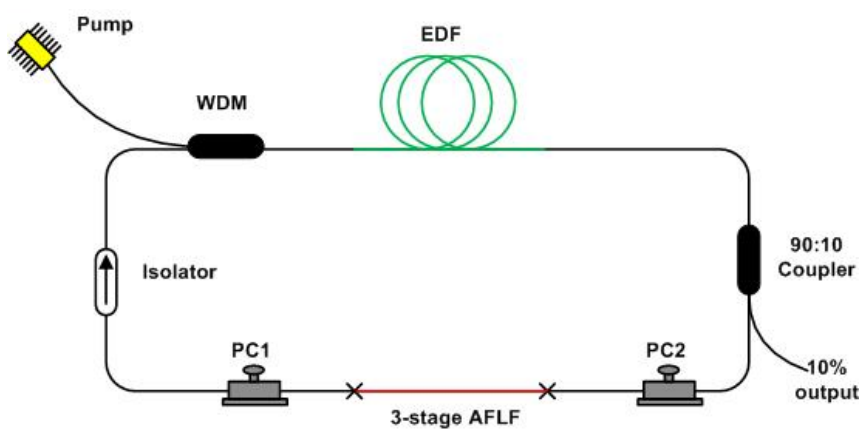

Fig. 3: The schematic of the Dual-wavelength fiber ring laser with an intra-cavity 3-stage AFLF.

The schematic of the proposed switchable dual-wavelength fiber ring laser is shown in Fig.3. A 3m long erbium doped fiber (EDF) with $18 \mathrm{~dB} / \mathrm{m}$ nominal absorption at $1530 \mathrm{~nm}$ is used as the gain material. The $975 \mathrm{~nm}$ laser diode pump source that could provide up to $300 \mathrm{~mW}$ pump power is employed through a 980/1550 wavelength division multiplexer (WDM). A 3-stage AFLF sandwiched between two polarization controllers (PCs) is used as wavelength selector. A 90:10 coupler is employed to couple $10 \%$ of the laser light out of the cavity. The unidirectional operation is achieved by applying a polarization independent isolator in the laser cavity.
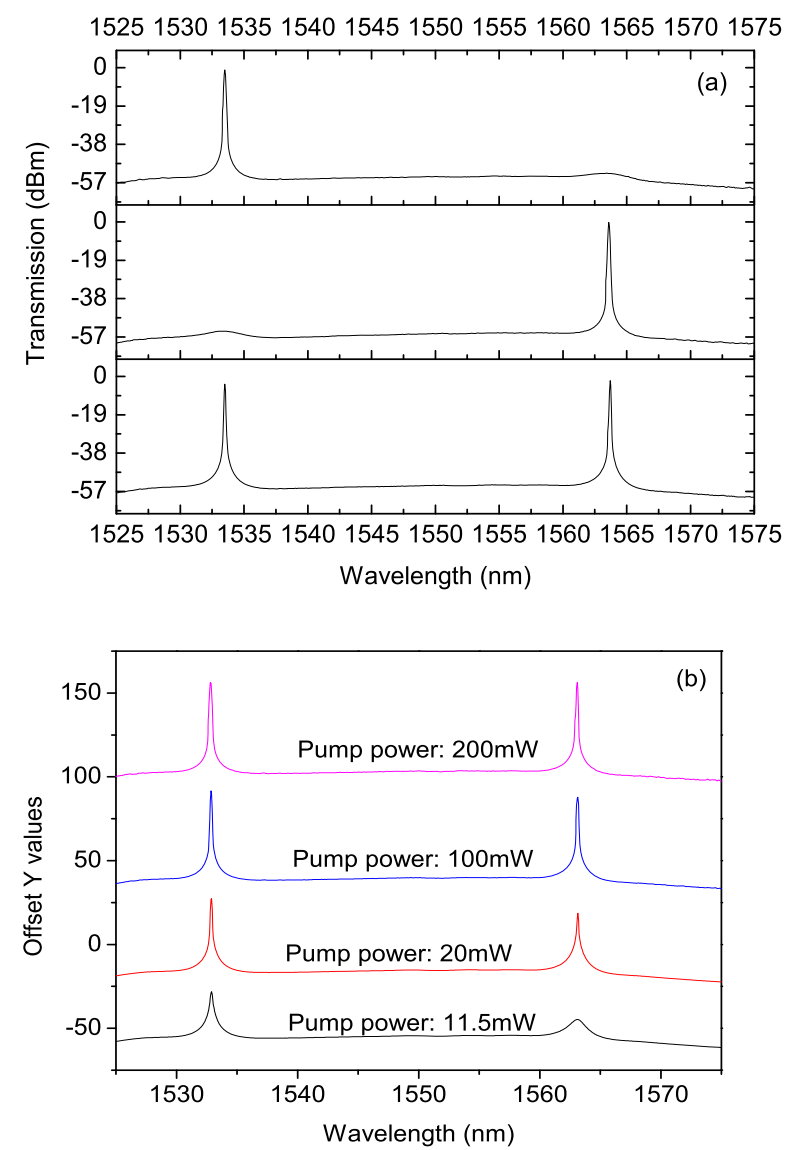

Fig. 4: Laser output spectra (a) at dual-wavelength and single wavelength operation and (b) dual-wavelength operation at different pump power.

In the experiment, the single or dual wavelength operation was obtained by adjusting the PCs. The output power level of each wavelength in dual-wavelength operation could also be adjusted by adjusting the PCs (see in Fig. 4 a). The pump power threshold of laser was around $11.5 \mathrm{~mW}$ for single wavelength operation and around $20 \mathrm{~mW}$ for dual-wavelength operation. The dual-wavelength operation could be only achieved at the suitable position of the PCs, however, once it was established, which could still self-started at next time. Fig. $4 \mathrm{~b}$ shows the output spectra of the ring laser with dual-wavelength operation, at different pump power. In the experiment, the laser was only excited at $1533.5 \mathrm{~nm}$ and $1563.3 \mathrm{~nm}$, and no other lasing modes were observed over the entire erbium gain band. The wavelength spacing of the dual wavelength laser is determined by the FSR of the 3-stage filter. 

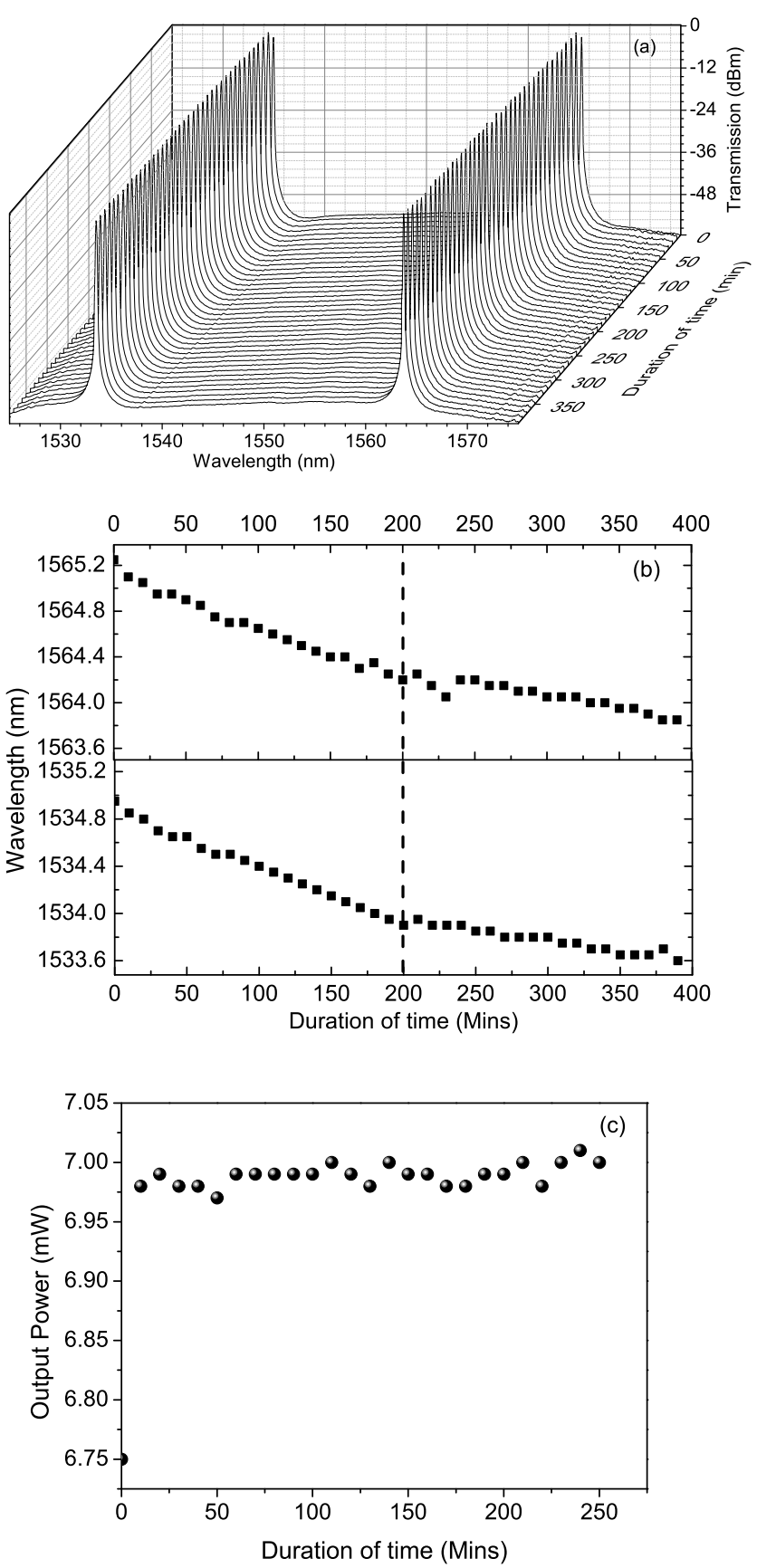

Fig. 5. Repeatedly scanned output spectra of the multi-wavelength laser: (a) dual-wavelength laser output and (b) wavelength drift with 10mins interval time, (c) the fluctuation of output power.

Fig. 5 (a) show laser output spectra captured with 10-min time intervals. The results show the 3-stage AFLF based switchable dual-wavelength laser is stable in terms of both lasing wavelength, which is attributed to the role of the 3-stage AFLF for its in-phase mode selecting function. As shown in Fig. 5 (b), the output wavelengths of laser were shifting to the shorter wavelength, which is because the temperature is increasing during experiment time, and the transmission band of Lyot filter is shifting to the short wavelength with increasing of temperature[28]. In Fig.5 b, it also shows there are two different slope regions for both wavelengths: at the first 200mins, the wavelength drifts were faster due to the cavity temperature was increased quickly. After 200mins, the temperature exchanging between fiber cavity and optical bench was getting equilibrium, and wavelength blue shift was because the temperature of environment was increasing. We monitored the output power at $200 \mathrm{~mW}$ pump power which the results was plotted in Fig 5c. As it shown, during 250mins with 10min time interval, the average power is around $6.99 \mathrm{~mW}$ with $0.02 \mathrm{~mW}$ fluctuation. The PERs of the output of the fiber laser at 1533.5nm and 1563.3nm are shown in Fig. 6 (a) and (b), which are at $34.6 \mathrm{~dB}$ and $34.5 \mathrm{~dB}$, respectively. Thus, the degree of polarization (DOP) of output laser at both wavelengths is higher than $99.9 \%$.
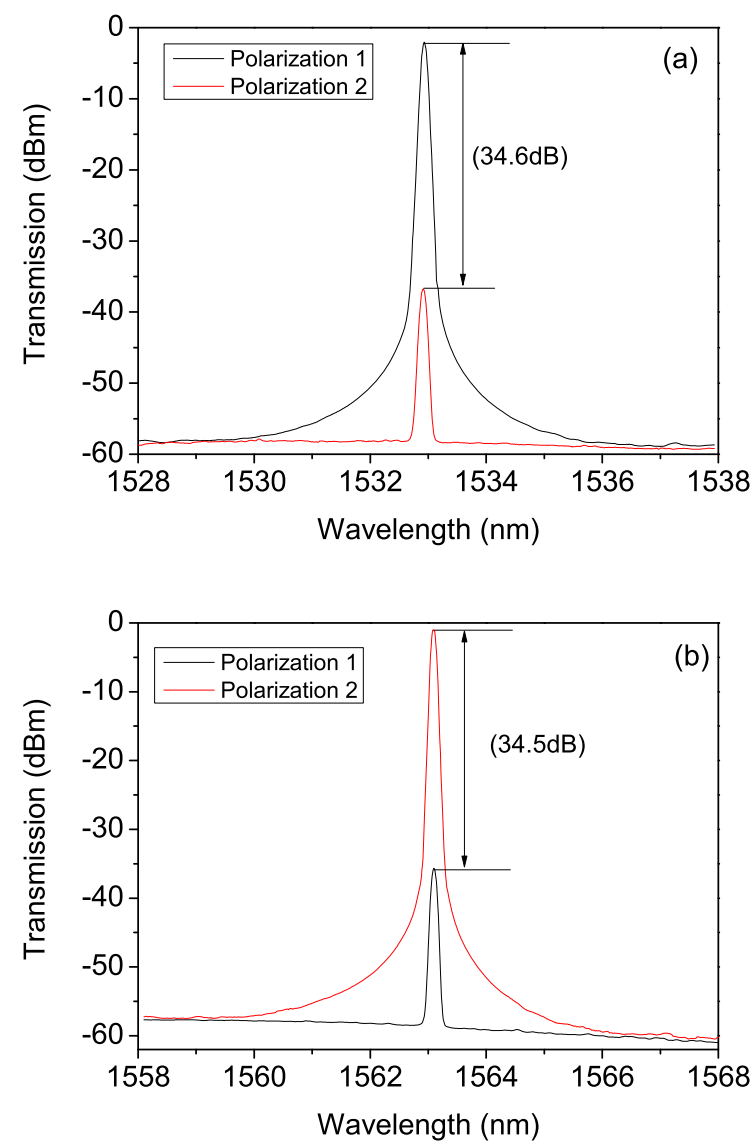

Fig. 6.The PER results of output laser at (a) $1533.5 \mathrm{~nm}$ and (b) $1563.3 \mathrm{~nm}$.

\section{CONCLUSION}

In this letter, we have reported a demonstration of a single polarization and switchable dual-wavelength EDFA based fiber ring laser system incorporating an intra-cavity 3-stage AFLF concatenating four $45^{\circ}$-TFGs separated by three PM fiber cavities of lengths at $20 \mathrm{~cm}, 40 \mathrm{~cm}$ and $80 \mathrm{~cm}$. The compact and integrated laser system shows a low pump threshold around $30 \mathrm{~mW}$ and stable output with low fluctuation on peak intensity and lasing wavelength due to the in-phase mode-selecting function of the multi-stage AFLF. The fluctuation of peak 
intensity and wavelength were only $0.02 \mathrm{~mW}$. Although the wavelength was shifting to shorter wavelength during experiment, we can stable the wavelength by applying temperature controller on the laser cavity. The output laser also shows very high PER of around $34 \mathrm{~dB}$, which indicates the laser output is at a high degree of single polarization.

\section{REFERENCES}

[1] L. R. Chen, "Tunable Multiwavelength Fiber Ring Lasers Using a Programmable High-Birefringence Fiber Loop Mirror," IEEE Photonics Technology Letters, vol. 16, pp. 410-412, 2004.

[2] Yu Yao, Xiangfei Chen, Yitang Dai, and Shizhong Xie, "Dual-wavelength erbium-doped fiber laser with a simple linear cavity and its application in microwave generation," IEEE Photonics Technology Letters, vol. 18, pp. 187-189, 2006.

[3] K. Venkataraayan, S. Askraba, K. E. Alameh, and C. L. Smith, "Multi-wavelength laser sensor for intruder detection and discrimination," Optics and Lasers in Engineering, vol. 50, pp. 176-181, 2012.

[4] B. I. Vasil'ev and O. Mannoun, "IR differential-absorption lidars for ecological monitoring of the environment," Quantum Electronics, vol. 36, pp. 801-820, September 30, 2006.

[5] S. Yamashita and K. Hotate, "Multiwavelength erbium doped fiber laser using intracavity etalon and cooled by liquid nitrogen," Electron. Lett., vol. 32, pp. 1298-1299, 1996.

[6] D. N. Wang, F. W. Tong, X. Fang, W. Jin, P. K. A. Wai, and J. M. Gong, "Multiwavelength erbium-doped fiber ring laser source with a hybrid gain medium," Opt. Commun., vol. 228, pp. 295-301, 2003.

[7] S. Qin, D. Chen, Y. Tang, and S. He, "Stable and uniform multi-wavelength fiber laser based on hybrid Raman and Erbium-doped fiber gains," Optics Express, vol. 14, p. 10522, 2006.

[8] S. Pan, C. Lou, and Y. Gao, "Multiwavelength erbium-doped fiber laser based on inhomogeneous loss mechanism by use of a highly nonlinear fiber and a Fabry-Pérot filter," Opt. Express, vol. 14, pp. 1113-1118, 2006.

[9] A. Bellemare, M. Karasek, M. Rochette, S. LaRochelle, and M. Tetu, "Room temperature multifrequency Erbium-doped fiber lasers anchored on the ITU frequency grid," J. Lightw. Technol., vol. 18, pp. 825-830, 2000.

[10]K. J. Zhou, D. Y. Zhou, F. Z. Dong, and N. Q. Ngo, "Room-temperature multiwavelength erbium-doped fiber ring laser employing sinusoidal phase-modulation feedback," Opt. Lett., vol. 28, pp. 893-895, 2003.

[11]J. Tian, Y. Yao, Y. Sun, X. Yu, and D. Chen, "Multiwavelength Erbium-doped fiber laser em- ploying nonlinear polarization rotation in a symmetric nonlinear optical loop mirror," Optics Express, vol. 17, p. 15160, August 17, 20092009

[12]X. H. Feng, H. Y. Tam, and P. K. A. Wai, "Stable and uniform multiwavelength erbium-doped fiber laser using nonlinear polarization rotation," Opt. Express, vol. 14, pp. 8205-8210, 2006.

[13]Y. Y. Zhu, W. L. Zhang, and Y. Jiang, "Tunable Multi-Wavelength Fiber Laser Based on Random Rayleigh Back-Scattering," IEEE Photonics Technology Letters, vol. 25, pp. 1559-1561, 2013.

[14]Q. Zhang, X. Zeng, F. Pang, M. Wang, and T. Wang, "Switchable multiwavelength fiber laser by using a compact in-fiber Mach-Zehnder interferometer," Journal of Optics, vol. 14, p. 045403, April 1, 20122012.

[15]X. Liu, L. Zhan, S. Luo, Z. Gu, J. Liu, Y. Wang, and Q. Shen, "Multiwavelength erbium-doped fiber laser based on a nonlinear amplifying loop mirror assisted by un-pumped EDF," Optics Express, vol. 20, p. 7088, March 26, 20122012

[16]G. Sun, D. S. Moon, A. Lin, W.-T. Han, and Y. Chung, "Tunable multiwavelength fiber laser using a comb filter based on erbium-ytterbium co-doped polarization maintaining fiber loop mirror," Optics Express, vol. 16, p. 3652, March 17, 20082008

[17]D. S. Moon, B. H. Kim, A. Lin, G. Sun, W.-T. Han, Y.-G. Han, and Y. Chung, "Tunable multi-wavelength SOA fiber laser based on a Sagnac loop mirror using an elliptical core side-hole fiber," Optics Express, vol. 15, p. 8371, 2007.

[18]Z. Zhang, L. Zhan, K. Xu, J. Wu, Y. Xia, and J. Lin, "Multiwavelength fiber laser with fine adjustment, based on nonlinear polarization rotation and birefringence fiber filter," Optics Letters, vol. 33, p. 324, 2008.

[19] Young-Geun Han, Gilhwan Kim, Ju Han Lee, Sang Hyuck Kim, and Sang Bae Lee, "Lasing wavelength and spacing switchable multiwavelength fiber laser from 1510 to $1620 \mathrm{~nm}$," IEEE Photonics Technology Letters, vol. 17, pp. 989-991, 2005.
[20]J. Yang, S. C. Tjin, and N. Q. Ngo, "Multiwavelength Tunable Fiber Ring Laser Based on Sampled Chirp Fiber Bragg Grating," IEEE Photonics Technology Letters, vol. 16, pp. 1026-1028, 2004.

[21] M. Li, X. Chen, T. Fujii, Y. Kudo, H. Li, and Y. Painchaud, "Multiwavelength fiber laser based on the utilization of a phase-shifted phase-only sampled fiber Bragg grating," Optics Letters, vol. 34, p. 1717, June 1, 20092009.

[22]C. Mou, P. Saffari, H. Fu, K. Zhou, L. Zhang, and I. Bennion, "Single- and dual-wavelength switchable erbium-doped fiber ring laser based on intracavity polarization selective tilted fiber gratings," Applied Optics, vol. 48, p. 3455, June 20, 20092009.

[23]Y. ÖHMAN, "A New Monochromator," Nature, vol. 141, pp. 157-158, January 22, 19381938.

[24]J. Evans, "The birefringent filter," J. Opt. Soc. Amer., vol. 39, pp. 229-242, 1949.

[25]Z. Yan, C. Mou, H. Wang, K. Zhou, Y. Wang, W. Zhao, and L. Zhang, "All-fiber polarization interference filters based on $45^{\circ}$-tilted fiber gratings," Optics Letters, vol. 37, p. 353, February 1, 20122012.

[26]M. Wang, S. Fu, P. Shum, N. Q. Ngo, J. Wu, and J. Lin, "A Tunable Lyot Birefringent Filter With Variable Channel Spacing and Wavelength Using Nonlinear Polarization Rotation in an SOA," IEEE Photonics Technology Letters, vol. 20, pp. 1527-1529, 2008.

[27]G. Shabtay, E. Eidinger, Z. Zalevsky, D. Mendlovic, and E. Marom, "Tunable birefringent comb filter-optimal iterative design," Opt. Express, vol. 10, pp. 1534-1541, 2002.

[28]Z. Yan, H. Wang, K. Zhou, Y. Wang, W. Zhao, and L. Zhang, "Broadband Tunable All-Fiber Polarization Interference Filter Based on 45\&\#176; Tilted Fiber Gratings," J. Lightwave Technol., vol. 31, pp. 94-98, 2013. 